\title{
Hydrothermalism Associeted To Ediacarian Volcanism Of Ait Sawn And Tissouktai (Eastern Anti- Atlas, Morocco)
}

\author{
R. Zahour \\ G. Zahour \\ Y. Zerhouni \\ S. Alikouss \\ Z. Baroudi \\ H. El Hadi
}

Laboratoire de Géodynamique des Chaines Anciennes, Université Hassa II de Casablanca, Faculté des Sciences, Ben M’Sik, Sidi Othmane, Casablanca, Maroc

doi: 10.19044/esj.2016.v12n24p316 URL:http://dx.doi.org/10.19044/esj.2016.v12n24p316

\begin{abstract}
In the South East of Ouarzazate (south west of the Saghro massif, Anti Atlas) outcrop the geological formations of Ait Sawn and Tissouktai. These, attributed to the Ediacaran (or terminal Neoproterozoic), consist of basic volcanic facies, intermediate and acid intercalated with pyroclastics and epiclastic levels. This set is traversed locally by acidic veins.

The rocks studied suffered intense weathering processes marked by the development of secondary minerals which are also formed in the hydrothermal veins and at the expense of the primary magmatic minerals and mesostasis. The main paragenesis of mineralisation consists of hematite, malachite, chalcocite, pyrite, barite, epidote, quartz, chlorite, calcite, albite, sericite and iron oxides.

Paleogeography sector is governed by inherited active faults of the Pan African base. Magmatism studied seems to be guided by these tectonic lineaments during the terminal Proterozoic extension. The reactivation of these tectonic structures, after the Cambrian, and probably during the Variscan and / or Atlasic, is certain.

Field investigations, supplemented by microscopic and geochemical study, indicate that much of the mineralization associated with volcanic Southwest Massif Saghro, is related to a hydrothermalisme contemporary with the stage of fault reactivation.
\end{abstract}


Keywords: Anti-Atlas, Saghro massif, Ediacaran, hydrothermalism, fault, mineralization

\section{Résumé}

Au Sud Est d’Ouarzazate (Sud-Ouest du massif de Saghro, Anti Atlas), affleurent les formations géologiques d’Ait Sawn et de Tissouktai. Ces dernières, attribuées à l’Ediacarien (ou Néoprotérozoïque terminal), sont constituées de faciès volcaniques (basiques, intermédiaires et acides) avec des intercalations de niveaux épiclastiques et pyroclastiques. Cet ensemble est parcouru localement par des filons acides. Les roches étudiées ont subi des processus d'altération intenses marqués par le développement de paragenèses secondaires qui se forment aussi bien dans les filons hydrothermaux qu'au dépend des minéraux primaires magmatiques et de la mésostase. L'association principale de la minéralisation est formée d’oligiste, hématite, malachite, chalcocite, pyrite, barytine, épidote, quartz, chlorite, calcite, albite et séricite. La paléogéographie du secteur est régie par des failles actives héritées du socle panafricain. Le magmatisme étudié semble être guidé par ces linéaments tectoniques pendant l'extension finiProtérozoïque. La réactivation de ces structures tectoniques, postérieurement au Cambrien, et probablement lors de l’orogenèse varisque et/ou atlasique, est certaine. Les investigations de terrain, complétées par l'étude microscopique et géochimique, indiquent qu'une grande partie des minéralisations associées aux volcanites du Sud-Ouest de la boutonnière de Saghro, est liée à un hydrothermalisme contemporain au stade de réactivation des failles.

Mots-clés : Anti-Atlas, Boutonnière de Saghro, Ediacarien, Hydrothermalisme, faille, minéralisation

\section{Introduction}

La synthèse des travaux de prospection minière, couplée aux résultats géochronologiques effectués sur les gisements du Maroc, ont permis de lier la genèse des minéralisations de l'Anti-Atlas à des événements hydrothermaux associés à des contextes géodynamiques bien définis, dont les meilleurs cibles semblent être associées au magmatismes calco-alcalins fortement potassique à alcalins d’âge édiacarien à Cambrien (Gasquet et Cheillets, 2009). En effet l'environnement géodynamique à cette époque correspond à un contexte extensif généralisé à l’échelle de l'Anti-Atlas qui a été propice à la genèse d’importantes minéralisations à teneurs économiques. Ce régime est accompagné d'un important flux thermique marqué par des gradients élevés, ce qui a favorisé la circulation de fluides hydrothermaux responsables de précipitations-reconcentrations des minéralisations 
polymétalliques disséminées dans le socle protérozoïque. Cette activité a affecté d'une manière très poussée les roches magmatiques.

Les boutonnières de Tissouktai et d'Ait Sawn, qui ont fait l'objet de cette étude, sont représentées par une épaisse série volcanique et volcanoclastique. Cette dernière garde encore l'empreinte de processus d’altération suggérant une importante activité hydrothermale qui représente une conséquence inévitable de la mise en place de perturbations thermiques dans un encaissant perméable et saturé en eau (Norton, 1978).

Un âge protérozoïque est généralement admis par de nombreux auteurs pour expliquer l’origine des minéralisations associées au volcanisme de cette époque (Leblanc, 1986 ; Abia et al., 1999 ; Baroudi et al., 1999 ; Mouttaqi et Sagon, 1999 ; Al Ansari et Sagon, 1999; Gasquet et al.,2005 et Belkacim, 2014). Il a été également admis que le transport de la minéralisation a été souvent assuré par des saumures complexes (En-nacri et al., 1997 ; Essaraj et al., 1998 et Baroudi, 2002).

Cette étude se fixe les objectifs suivants :

- caractériser le phénomène d’hydrothermalisme et les minéralisations associées aux paléovolcanites édiacariennes étudiées à travers une étude à différentes échelles d'observations (affleurement, microscope et géochimie);

- préciser le contrôle et l'impact de la tectonique sur l’origine des minéralisations ;

- discuter l’âge des minéralisations.

\section{Cadre géologique}

Les boutonnières de Tissouktai et d'Ait Sawn se situent respectivement à 34 et $40 \mathrm{~km}$ au SE de la ville d'Ouarzazate et constituent la partie occidentale du massif de Saghro (Figure 1a et 1b). Elles correspondent à des bombements disposés suivant une direction NE-SW. Ces structures comportent un socle d'âge Ediacarien constitué par des roches volcaniques et volcanoclastiques recouvert en discordance par une couverture schisto-grésocarbonatée du Cambrienne et adoudounnienne (Zahour et al., 2014).

Les datations des coulées volcaniques dans la région d’Ouarzazate ont fourni des âges compris entre $586 \pm 20$ et $563 \pm 10$ Ma (Mifdal et Peucat, 1985). Les ignimbrites de cette région ont livré un âge de $567 \pm 7 \mathrm{Ma}$ (Walsh et al., 2002). Ces valeurs permettent d'attribuer les roches étudiées au Précambrien III selon Choubert (1963) ou au Néoprotérozoïque terminal (Plumb, 1991), ou encore au super groupe d'Ouarzazate selon la classification de Thomas et al. (2004). 


\section{Lithostratigraphie et pétrographie}

La cartographie détaillée et la corrélation des coupes sériées réalisées au niveau des séries de l'Ediacarien des boutonnières de Tissouktai et d'Ait Sawn, ont permis d'établir une succession lithostratighraphique synthétique propre à chaque boutonnière (Figure 2) :

\section{Ediacarien de la boutonnière d'Ait Sawn}

La série débute par des brèches pyroclastiques $(30 \mathrm{~m})$ qui correspondent au faciès de base de la série étudiée. Il s'agit de projections violentes qui annoncent les premières venues magmatiques du secteur. Les brèches sont surmontées par une succession de coulées métriques de basaltes et d'andésites basiques qui occupent une grande étendue. Ces coulées montrent une diversité de faciès, dont le plus dominant est celui d'une texture porphyrique à mégaporphyrique vacuolaire. Ces faciès sont parcourus de fions d'épidote et de filons de quartz auquel est associé souvent l'oligiste. L'ensemble basique et intermédiaire passe à un ensemble volcanoclastique composite $(45 \mathrm{~m})$ représenté par des brèches pyroclastiques, des conglomérats épiclastiques et des alternances de niveaux centimétriques gréseux et bréchiques. La partie sommitale de cet ensemble correspond à une période de sédimentation qui marque un arrêt momentané de l'activité volcanique. L'épisode volcanosédimentaire est interrompu par un second épisode magmatique représenté par des coulées basaltiques (20 m) de couleur grisâtre à sombre, presque aphyrique à l'œil nu.

Le troisième épisode volcanique correspond à des émissions acides marquées par la mise en place d'une puissante série ignimbritique fluidale et nodulaire $(160 \mathrm{~m})$.

Le magmatisme filonien est représenté par un filon rhyolitique d'une puissance de 10 à $30 \mathrm{~m}$ et d'une extension de plusieurs centaines de mètres. Ce filon a une direction N60 et un pendage subvertical. Il est encaissé tantôt dans les brèches pyroclastiques de base tantôt dans les basaltes et représente une voie d'alimentation probable du magmatisme acide du secteur.

Sur la série volcanique et volcanoclastique étudiée, repose en discordance, une puissante couverture cambrienne et adoudounienne schistogréso-conglomératique et carbonatée. 


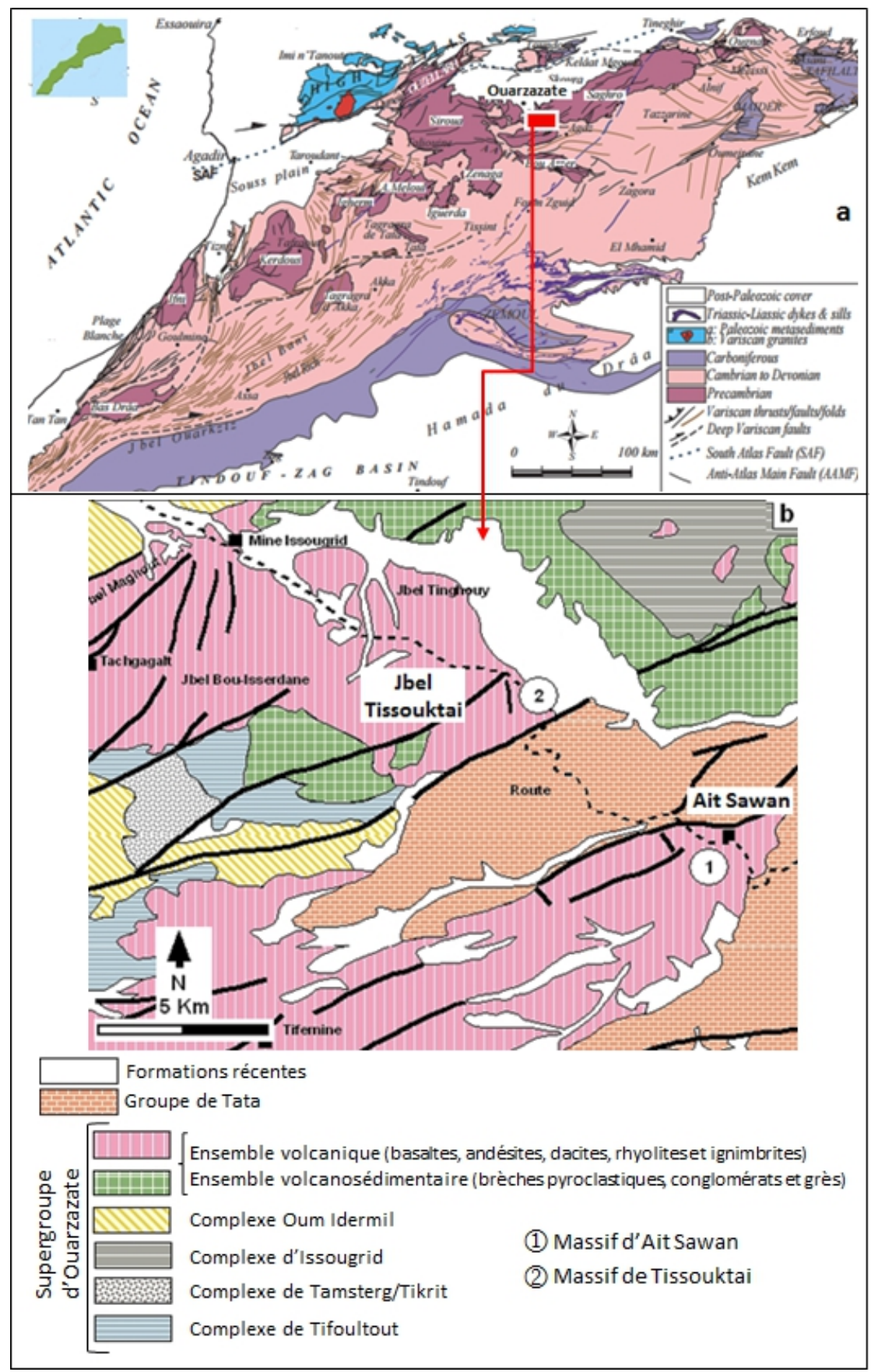

Figure 1 : (a) Schéma géologique de l'Anti-Atlas marocain avec localisation des boutonnières précambriennes (Choubert, 1963), (b) Carte géologique de la partie occidentale du massif de Saghro avec localisation des boutonnières étudiées (Alvaro et al., 2010, modifiée). 


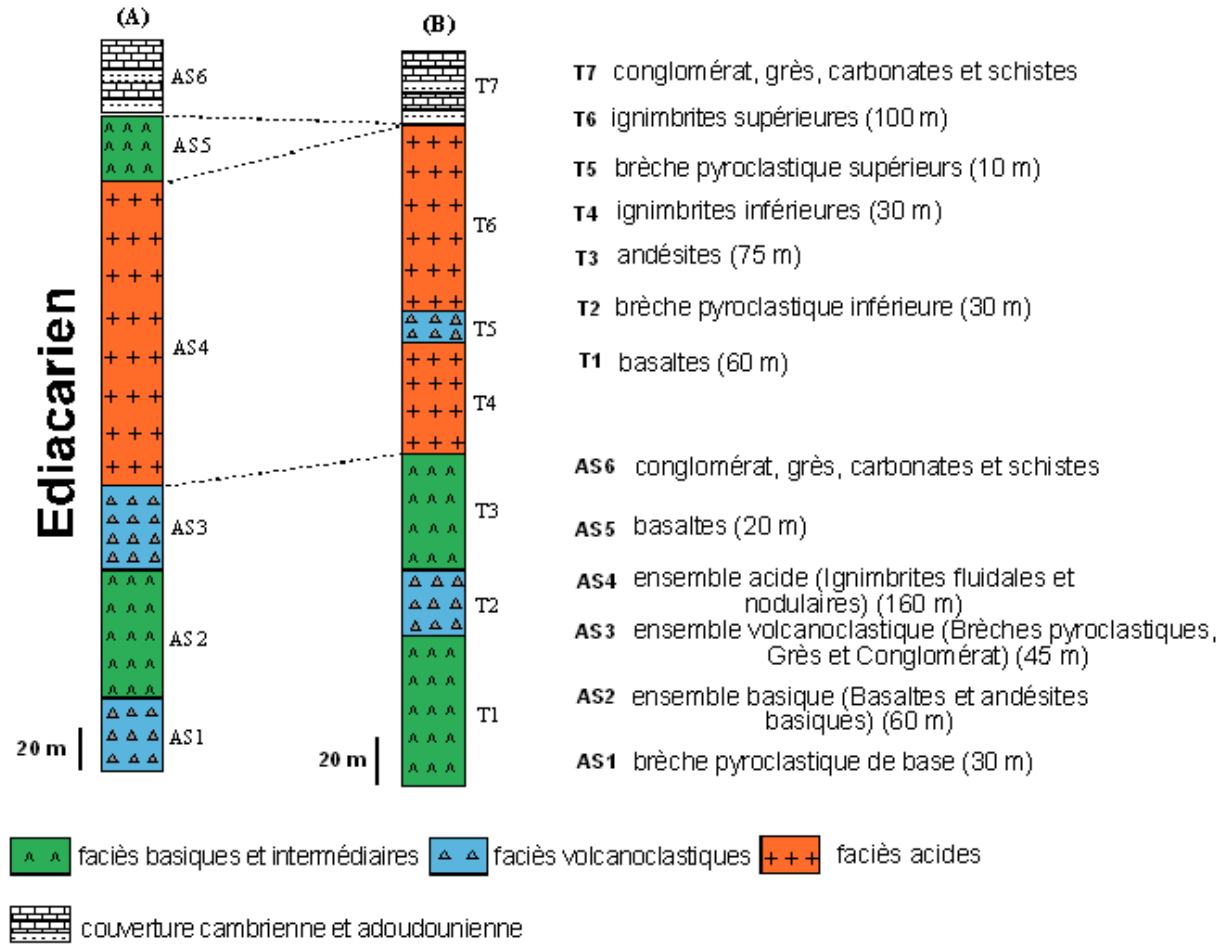

Figure 2 : Succession lithostratigraphique de l'Ediacarien des boutonnières d'Ait Sawn (A) et de Tissouktai (B).

\section{Ediacarien de la boutonnière de Tissouktai}

La succession lithostratigraphique commence par des basaltes (60 m), qui s'organisent en plusieurs coulées gris-verdâtre fortement altérées. Les basaltes présentent localement des zones métriques épidotisées et un réseau filonien subméridien à N60 de quartz ou de barytine, oligiste et hématite. Ces coulées passent aux brèches pyroclastiques inférieures qui forment un niveau décamétrique (20 à $30 \mathrm{~m}$ ), dessinant une bande séparant les basaltes des autres formations sus-jacentes. L'épisode volcanoclastique est suivi par un autre épisode volcanique marqué par des roches andésitiques $(75 \mathrm{~m})$. Les andésites sont surmontées par les brèches pyroclastiques supérieures qui forment le faciès de transition entre l'ensemble précédent essentiellement basique et intermédiaire, et l'ensemble sus-jacent acide. Ce dernier est essentiellement ignimbritique et formé de deux faciès :

- ignimbrites inférieures vitroclastiques et xénolitiques $(30 \mathrm{~m})$, sombres sur patine et rouge brique sur cassure fraiche. A l'œil nu, on peut observer des enclaves plus sombres, de forme anguleuse; du quartz globulaire et des structures de différentes formes remplies de quartz (vitroclastes) bien visible sur cassure ; 
- $\quad$ ignimbrites supérieures formées d’empilement de coulées (100m) à xénolites et à sphérolites.

Les ignimbrites supérieures de Tissouktai sont en contact faillé avec la couverture cambrienne et adoudounienne par l'intermédiaire de l'accident de Tissouktai qui est matérialisé par un filon d’épaisseur décamétrique de quartz minéralisé, orienté N60, et qui s’étend sur $2 \mathrm{Km}$.

Le magmatisme filonien est représenté par un filon rhyolitique d'une puissance de $15 \mathrm{~m}$ et d'une extension de plusieurs centaines de mètres. Ce filon, de direction NS, a un pendage vertical. Il est encaissé entre les brèches pyroclastiques inférieures et les andésites.

L’étude microscopique effectuée sur plusieurs échantillons permet de mettre en évidence une diversité de faciès, dont les principaux caractères pétrographiques des roches étudiées sont résumés dans le tableau 1.

\begin{tabular}{|c|c|c|c|c|c|c|c|}
\hline \multirow[t]{2}{*}{$\begin{array}{l}\text { Bouton } \\
\text { nière }\end{array}$} & \multirow{2}{*}{$\begin{array}{l}\text { Nature de } \\
\text { la roche }\end{array}$} & \multirow{2}{*}{ Faciès } & \multirow{2}{*}{$\begin{array}{l}\text { Paragenèse } \\
\text { primaire }\end{array}$} & \multirow{2}{*}{$\begin{array}{l}\text { Paragenèse } \\
\text { secondaire }\end{array}$} & \multicolumn{2}{|c|}{$\begin{array}{c}\text { \% des } \\
\text { Constituants }\end{array}$} & \multirow{2}{*}{ Texture } \\
\hline & & & & & $\begin{array}{c}\text { Crista } \\
\text { ux }\end{array}$ & $\begin{array}{l}\text { Méso } \\
\text { stase }\end{array}$ & \\
\hline \multirow{11}{*}{$\begin{array}{c}\text { Ait } \\
\text { Sawn }\end{array}$} & \multirow{3}{*}{ Basaltes } & Faciès à olivine et pyroxène & $\begin{array}{l}\mathrm{Pl}, \mathrm{Px}, \mathrm{Ol} \\
\text { et Op }\end{array}$ & $\begin{array}{c}\text { Ser, Chl, Ep, Qz, } \\
\text { Al et Oxy }\end{array}$ & $80 \%$ & $20 \%$ & Microlitique porphyrique \\
\hline & & Faciès fluidal et vacuolaire & $\begin{array}{l}\text { Pseudomor } \\
\text { phes de } \\
\text { Ol, } \mathrm{Pl}, \mathrm{Px} \\
\text { et Op }\end{array}$ & Chl et Oxy & $10 \%$ & $90 \%$ & $\begin{array}{l}\text { Microlitique porphyrique } \\
\text { fluidale et vacuolaire }\end{array}$ \\
\hline & & $\begin{array}{c}\text { Faciès microlitique } \\
\text { porphyrique et vacuolaire }\end{array}$ & Pl et Op & $\begin{array}{l}\text { Qz, Cal, Ep, Al et } \\
\text { Oxy }\end{array}$ & $70 \%$ & $30 \%$ & $\begin{array}{l}\text { Microlitique porphyrique } \\
\text { vacuolaire }\end{array}$ \\
\hline & $\begin{array}{c}\text { Andésites } \\
\text { Basiques }\end{array}$ & $\begin{array}{c}\text { Faciès Microlitique } \\
\text { porphyrique vacuolaire }\end{array}$ & $\mathrm{Pl}$ et $\mathrm{Op}$ & $\begin{array}{l}\text { Cal, Fld, Qz et } \\
\text { Oxy }\end{array}$ & $40 \%$ & $60 \%$ & $\begin{array}{l}\text { Microlitique porphyrique } \\
\text { vacuolaire }\end{array}$ \\
\hline & \multirow{3}{*}{ Ignimbrites } & Faciès fluidal et rubané & Qz et Op & Qz et Oxy & $5 \%$ & $95 \%$ & Eutaxitique \\
\hline & & Faciès sphérolitique & Qz et Op & Qz et Oxy & $5 \%$ & $95 \%$ & Vitroclastique sphérolitique \\
\hline & & $\begin{array}{c}\text { Faciès rubané et } \\
\text { sphérolitique }\end{array}$ & Qz et Op & Qz et Oxy & $5 \%$ & $95 \%$ & Rubanée \\
\hline & Rhyolites & Cryptocristallin & $\begin{array}{l}\text { Qz et Fld } \\
\text { et Op }\end{array}$ & Qz et Oxy & $17 \%$ & $83 \%$ & Cryptocristalline \\
\hline & $\begin{array}{c}\text { Brèches } \\
\text { pyroclastique } \\
\text { s } \\
\end{array}$ & --------- & \multicolumn{5}{|c|}{ Fragments de roches (85\%), Pl et Op (5\%) et Ciment (10\%) } \\
\hline & Basaltes & & $\begin{array}{c}\mathrm{Pl} \text {, Ol et } \\
\text { Op }\end{array}$ & Chl et Cal & $40 \%$ & $60 \%$ & microlitique porphyrique \\
\hline & \multirow[b]{2}{*}{ Andésites } & $\begin{array}{c}\text { Faciès microlitique } \\
\text { porphyrique fluidale }\end{array}$ & $\begin{array}{c}\mathrm{Pl}, \mathrm{Ol} \text { et } \\
\mathrm{Op}\end{array}$ & Oxy & $32 \%$ & $68 \%$ & $\begin{array}{l}\text { microlitique porphyrique } \\
\text { fluidale }\end{array}$ \\
\hline \multirow[t]{4}{*}{$\begin{array}{l}\text { Tissouk } \\
\text { tai }\end{array}$} & & $\begin{array}{l}\text { Faciès microlitique } \\
\text { porphyrique à pyroxènes et } \\
\text { fantômes d'olivines }\end{array}$ & $\begin{array}{l}\mathrm{Pl}, \mathrm{Px}, \mathrm{Ol} \\
\quad \text { et Op }\end{array}$ & Chl et Oxy & $50 \%$ & $50 \%$ & microlitique porphyrique \\
\hline & \multirow[b]{2}{*}{ Ignimbrites } & $\begin{array}{c}\text { Faciès vitroclastique } \\
\text { xénolitique }\end{array}$ & $\begin{array}{c}\text { Qz, Pl, } \\
\text { Fld, Op et }\end{array}$ & Cal, Mus et Oxy & $50 \%$ & $50 \%$ & vitroclastique xénolitique \\
\hline & & Faciès eutaxitique & $\begin{array}{l}\text { Qz, Fld, } \\
\text { Op et } \\
\text { fragments } \\
\text { de roches }\end{array}$ & $\begin{array}{l}\text { Cal, Ser, Mus et } \\
\text { Oxy }\end{array}$ & $30 \%$ & $70 \%$ & Eutaxitique \\
\hline & $\begin{array}{c}\text { Brèches } \\
\text { pyroclastique } \\
\text { s }\end{array}$ & --- & \multicolumn{5}{|c|}{ Fragments de roches (50\%), Chl, Pl, Mus Op (26\%) et Ciment (24\%) } \\
\hline
\end{tabular}

Tableau 1 : Tableau récapitulatif de la pétrographie des volcanites de la série du

Néoprotérozoïque terminal des boutonnières d'Aït Sawn et de Tissouktai. Abréviations : Pl :

plagioclase, Px : pyroxène, Ol : olivine, Op : opaques, Qz : quartz, Fld : feldspaths, Mus :

muscovite, $\mathrm{Al}$ : Albite, Ep : épidote, Cal : calcite, Ser : séricite, Chl : chlorite et Oxy : oxydes. 


\section{Altérations hydrothermales et minéralisations}

Les interactions entre l'encaissant et les fluides hydrothermaux se traduisent par l'apparition d'importantes phases d'altérations à Tissouktai et à Ait Sawn. Ces transformations se manifestent à toutes les échelles (terrain, observation microscopique et analyses géochimiques).

\section{A l'échelle du terrain}

A l'échelle de l'affleurement, les phénomènes d’altération se traduisent par des habitus variées: barytine ;

filons, filonnets et veines à quartz, oligiste, hématite, épidote et

- $\quad$ vacuoles à feldspath, chlorite, calcite et quartz (Planche 1. a) ;

- $\quad$ géodes à quartz et sphérolites à calcédoine et quartz.

Deux principaux indices enregistrés sur le terrain permettent de soutenir la présence d'une importante silicification :

(1) Présence de nombreuses vacuoles remplies de quartz dans les andésites d’Ait Sawn ainsi que les géodes de quartz (Planche 1. b) observées dans les basaltes sommitaux du même secteur. Il en est de même pour les ignimbrites du massif d'Ait Sawn et à moindre celles de Tissouktai qui renferment des nodules siliceux qui peuvent atteindre $15 \mathrm{~cm}$ de diamètre.

(2) Développement de nombreuses veines, filonnets et filons de quartz. Ces derniers sont d'ordres centimétriques à Ait Sawn et métriques à Tissouktai, ils sont encaissés dans toute la série du Néoprotérozoïque Terminal. Ces filons de quartz sont tantôt stérile tantôt associés aux minéralisations ferrifères ou cuprifères. La silicification semble être régionale, puisqu’elle a été observée dans les deux boutonnières.

Un autre phénomène enregistré sur le terrain, est marqué par le développement de l'épidote qui se rencontre uniquement dans les basaltes inférieurs du massif d'Ait Sawn et de Tissouktai (Planche 1.c).

La minéralisation ferrifère est marqué sur le terrain par la présence de filons mixtes à quartz, oligiste et l'hématite orientés NS et N170 et par des filons à oligiste seul de direction N60. Ces structures subverticales, à puissance inframétrique et d'extensions plurimétriques $(<30 \mathrm{~m})$, sont encaissées dans les basaltes et les andésites de Tissouktai et d'Ait Sawn. Dans les filons mixtes, le quartz tapisse les périphéries et présente un aspect bréchique dont les fragments sont souvent emballés dans l'oligiste qui occupe le cœur des filons (Planche 1. d). Cette observation suggère que la minéralisation ferrifère s'est mise en place lors d'une importante phase de bréchification postérieure au dépôt du quartz.

Une autre génération de minéralisation filonienne, à puissance centimétrique et à extension pluridécimétriques à barytine et oligiste a été rencontrée dans la boutonnière de Tissouktai. Ces filonnets sont orientés 


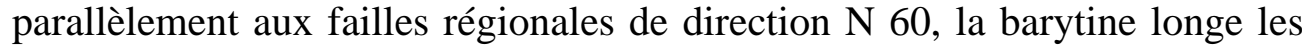
épontes alors que l'oligiste occupe le cœur des filons. La barytine se présente soit en rosettes cristallisées, de couleur rose, soit en cristaux lamellaires blanchâtres. Les relations texturales indiquent la précocité de la barytine par rapport à l'oligiste.

Dans le massif de Tissouktai, la carbonatation se matérialise par le développement de la malachite dans un grand filon subvertical de quartz orienté N60 et s'étend sur $2 \mathrm{~km}$ de longueur pour une puissance pouvant atteindre localement le décamètre. Il épouse la faille qui souligne le contact entre la série de l'Ediacarien du massif de Tissouktai et la couverture cambrienne et adoudounienne (Planche 1. e). La minéralisation est représentée par des sulfures, des carbonates de cuivre et des silicates de cuivre hydroxylés (chrysocole) (Planche 1. f).

\section{A à l'échelle microscopique}

Les roches étudiées ont subi des processus d'altération intenses marqués par le développement de minéralisations secondaires qui se forment aussi bien dans les vacuoles qu'au dépend des minéraux primaires magmatiques et de la mésostase.

\section{Etudes des vacuoles}

Dans les faciès basiques et intermédiaires d'Ait Sawn et de Tissouktai, la mésostase inclut localement des vacuoles millimétriques très variées, tant par la nature des minéraux secondaires qui les ont colmatées (feldspaths, quartz et calcite), que par leurs formes (arrondie, ovoïde, amiboïde et à contour courbé) et leurs tailles. Ces structures peuvent êtres isolées ou communicantes, ce qui témoigne de la richesse du magma en gaz et de sa très grande mobilité dans la lave (Fig. 3).

Les vacuoles enregistrent la circulation des fluides hydrothermaux. Deux types de remplissage peuvent être distingués :

(1) Monominéral : composé de feldspaths ou de calcite ;

(2) Séquentielle, qui montre soit :

- des vacuoles à feldspath au niveau des parois et à silice au cœur (Planche 2. a) ;

- $\quad$ des vacuoles à feldspath au niveau des parois et à quartz au cœur ;

- $\quad$ des vacuoles à bordure de calcite englobant un noyau de silice.

La disposition des minéraux tapissant les vacuoles permet de déduire deux principaux stades de remplissage :

- $\quad$ cristallisation des feldspaths en bordure des vacuoles ;

- $\quad$ remplissage par de la calcite provenant probablement de l'altération des plagioclases calcique, ... 


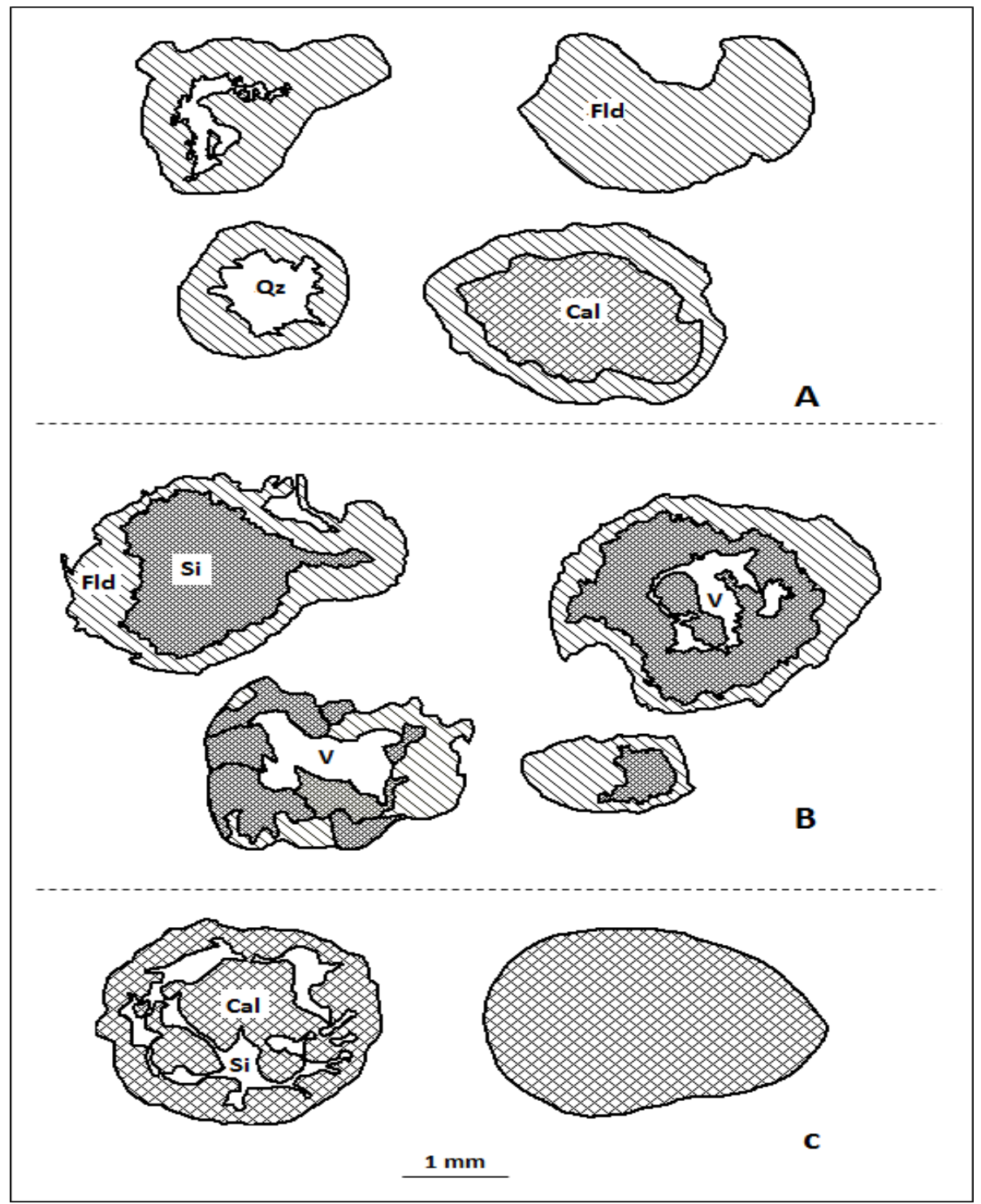

Figure 3: Formes et types de remplissage des vacuoles dans les roches étudiées:

(A) : Andésites basiques d’Ait Sawn (Fld : Feldspath et Qz : Quartz) ; (B) Basaltes inférieurs d'Ait Sawn ((Fld : Feldspath, Sl : Silice fibreuse et V : Vide) ; (C) : Basaltes sommitaux d'Ait Sawn (Cal : Calcite et Si : Silice amorphe).

\section{Etude des transformations minéralogiques secondaires et phénomènes associés}

Dans les roches volcaniques étudiées, l'ensemble des minéraux primaires a été le siège de nombreuses transformations secondaires plus ou moins importantes en relation avec la circulation de fluides hydrothermaux. Ces transformations se traduisent au microscope par le développement d'une 
paragenèse secondaire à albite, quartz, séricite, chlorite, calcite, épidote et oxydes de fer (Tab. 2) :

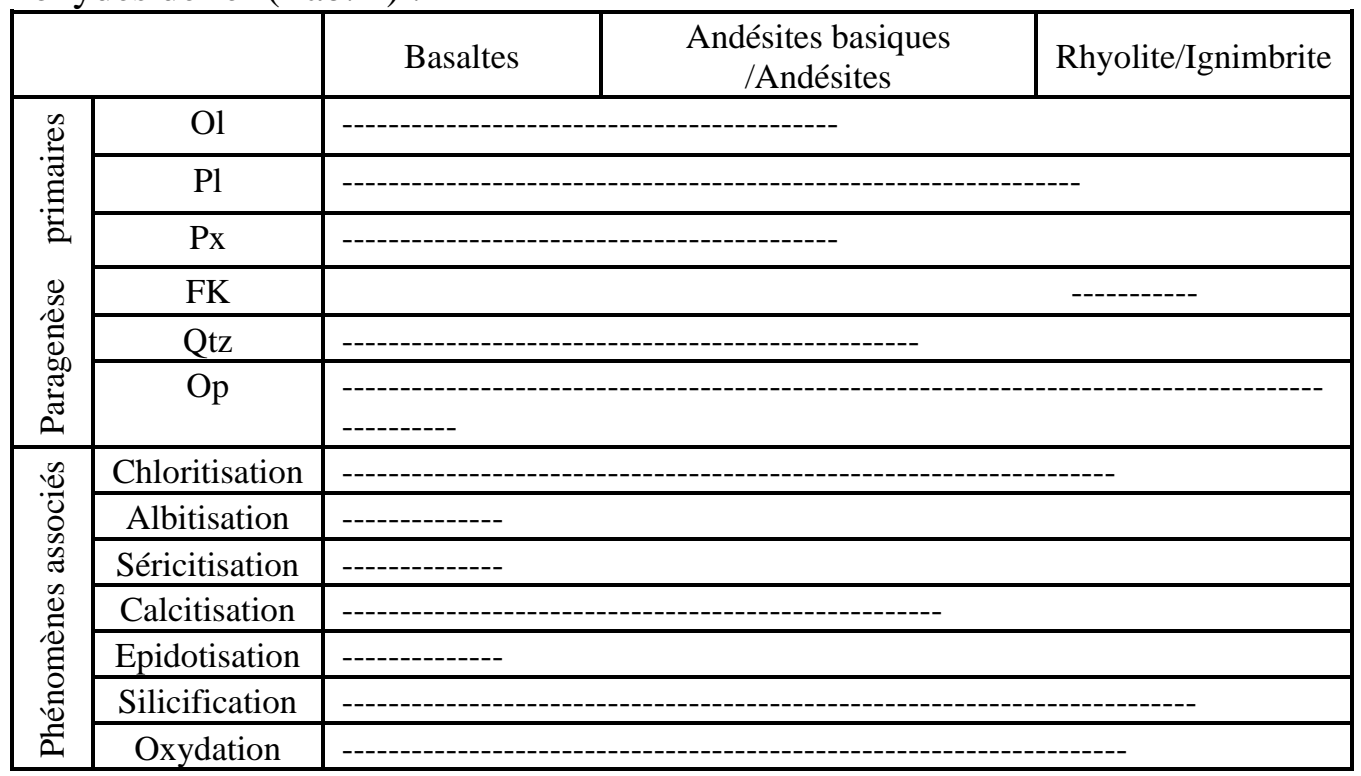

Tableau 2 : Répartition des minéraux primaires et phénomènes d’altération associés.

Les roches étudiées ont été affectées par différents phénomènes hydrothermaux qui ont provoqué notamment :

\section{Séricitisation}

Elle affecte partiellement ou totalement les phénocristaux de plagioclases (Planche 2. b) où elle se présente sous forme de fines paillettes et rarement en petites plages disséminées dans la matrice cryptocristalline. Il est probable que les roches basiques soient envahies par un fluide riche en potassium lié vraisemblablement à un hydrothermalisme métasomatique potassique.

\section{Chloritisation}

La chlorite se développe au détriment des minéraux ferromagnésiens (Figure 4) dans les roches basiques et intermédiaires.

Ces chlorites polarisent dans des teintes anormales bleuâtres (Planche 2. b) et rappellent celles analysées par Zahour (1990) des faciès identiques de la boutonnière de Siroua et correspondraient à la penninite (très riche en Mg). La limite supérieure de stabilité de ce type de chlorites est de l'ordre de $700^{\circ} \mathrm{C}$ et sous une pression totale de l'ordre de $2 \mathrm{~kb}$ (Fawcett et Yoder, 1966). 


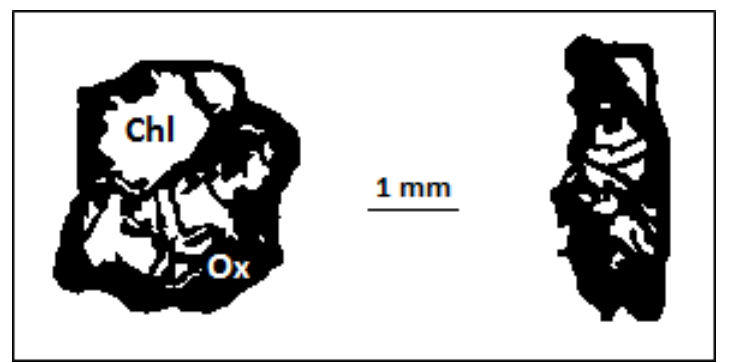

Figure 4 : Aspects des fantômes d’olivines entièrement altérées en chlorites et oxydes dans les basaltes d'Ait Sawn.

\section{Silicification et dévitrification}

La silicification offre des remplissages et des textures très variés (vacuoles, veines, ...). Les faciès acides sont caractérisés par des textures sphérolitiques (Planche 2. c).

La dévitrification qui affecte partiellement la pâte vitreuse de certaines ignimbrites se traduit par un fond felsitique microristallin quartzeux. Ce mécanisme se produit lors de la mise en place du magma ignimbritique riche en gaz, et il se poursuit même après sont refroidissement. Les éléments volatils associés à ce magma (hydrothermalisme tardimagmatique) en circulant dans les interstices provoqueront une recristallisation du verre volcanique.

\section{Calcitisation}

Outre son apparition dans certaines vacuoles, elle affecte les plagioclases dans les basaltes sommitaux d'Ait Sawn (Figure 5). La présence ou l'absence de la calcite dans un assemblage hydrothermal reflète la concentration du $\mathrm{CO}_{2}$ dans le fluide aqueux (GIGGENBACH, 1989). L'altération des plagioclases en calcite dans les basaltes peut être expliquée par la réaction suivante: Plagioclase calcique $+\mathbf{C O}_{2} \rightarrow$ Calcite + minéraux argileux.

La calcite affecte également les minéraux ferromagnésiens et la mésotase dans les basaltes de Tissouktai.

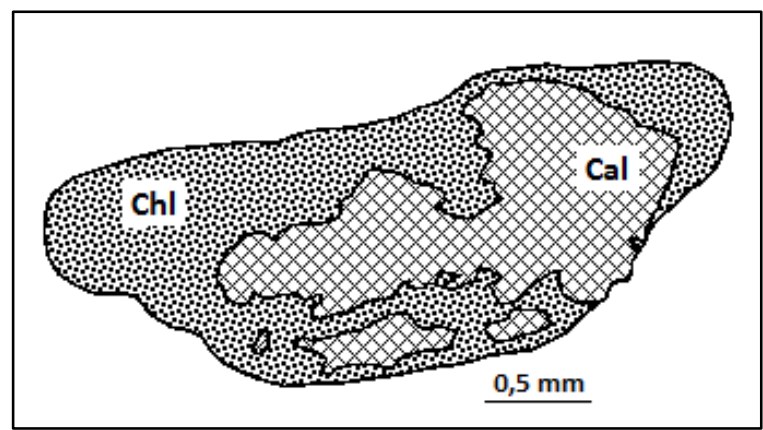

Figure 5 : Habitus d’un ferromagnésiens, altéré en calcite (Cal) et chlorite (Chl) (basaltes de Tissouktai). 


\section{Albitisation}

Elle affecte surtout les basaltes d'Ait Sawn et se matérialise par l'apparition de fines paillettes d'albite dans les plagioclases. Rosenbaueur et al., (1988), suggère qu'en plus d'un apport en sodium il faut un excès en silice dans le milieu pour transformer les plagioclases calciques en albite $\left(2 \mathrm{Na}^{+}+\mathrm{CaAl}_{2} \mathrm{Si}_{2} \mathrm{O}_{8}+4 \mathrm{SiO}_{2} \rightarrow 2 \mathrm{NaALSi}_{3} \mathrm{O}_{8}+\mathrm{Ca}^{2+}\right)$. Le calcium libéré peut s'associer avec le $\mathrm{CO}_{2}$ et précipite sous forme de carbonates. Dans notre cas, cette association est exprimée sous forme de calcite dans les vacuoles.

\section{Epidotisation}

Cette altération touche essentiellement les pyroxènes et des plagioclases calciques des basaltes. La transformation des plagioclases en épidote à $\mathrm{BT}^{\circ} \mathrm{C}$ exige une $\mathrm{fO}_{2}$ élevée (Liou, 1973) et elle se déroule comme suit : $4 \mathrm{CaAl}_{2} \mathrm{Si}_{2} \mathrm{O}_{8}+\mathrm{H}_{2} \mathrm{O} \rightarrow 2 \mathrm{Ca}_{2} \mathrm{AL}_{3} \mathrm{Si}_{3} \mathrm{O}_{12}(\mathrm{OH})+2 \mathrm{SiO}_{2}+\mathrm{AL}_{2} \mathrm{O}_{3}$

\section{Oxydation}

Elle se rencontre dans les faciès basiques et intermédiaires où on assiste à un remplacement des minéraux ferromagnésiens par des oxydes de fer (Figure 3 et Planche 2. b). Cette oxydation se manifeste également sous forme de filons d'oligiste et d'hématite.

\section{A l'échelle géochimique}

Dans le but de définir l'influence des solutions aqueuses sur le comportement de certains éléments majeurs (Alcalins, $\mathrm{SiO}_{2}, \mathrm{Fe}_{2} \mathrm{O}_{3}$ et $\mathrm{MgO}$, $\mathrm{CaO}, \mathrm{K}_{2} \mathrm{O}$ et $\mathrm{Na}_{2} \mathrm{O}$ ), on a dressé les diagrammes d'oxydes en fonction de la perte au feu (Figure 6). Il est admis que les pertes au feu augmentent naturellement en fonction du degré d’altération, par conséquent, la distribution des différents oxydes dans ces diagrammes nous permettra de quantifier et d’évaluer les différents processus d'altération ayant affecté les volcanites étudiées.

En effet, le diagramme $\mathrm{SiO}_{2}$ vs PF indique une corrélation négative. Cette distribution pourrait être la conséquence d'un hydrothermalisme responsable de la mobilité et le lessivage de $\mathrm{SiO}_{2}$ comme en témoigne les phénomènes de silicification enregistrés.

Le diagramme $\mathrm{CaO}$ vs $\mathrm{PF}$ montre une corrélation positive et s'explique par un apport du Ca, comme l'atteste d'une part, la calcitisation des plagioclases et de la mésostase, et d'autre part, le remplissage des vacuoles par de la calcite. Le $\mathrm{MgO}$ et le $\mathrm{Fe}_{2} \mathrm{O}_{3}$ présentent la même corrélation. Celle du $\mathrm{MgO}$ indique sa mobilité et se traduit par la néoformation des chlorites magnésiennes dans les roches basiques. $\mathrm{Le}^{\mathrm{Fe}} \mathrm{O}_{2} \mathrm{O}_{3}$ pourrait être lié à un apport en fer qui est marqué par le développement d'un réseau de filons à oligiste et hématite. 
Le $\mathrm{Na}_{2} \mathrm{O}$ vs $\mathrm{PF}$ présente une très faible corrélation positive et pourrait s'expliquer par une contribution du $\mathrm{Na}$ marquée par l'albitisation des plagioclases calciques.

Le diagramme de $\mathrm{K}_{2} \mathrm{O}$ vs $\mathrm{PF}$ met en évidence une corrélation négative qui indique que les phénomènes d'altération s'accompagnent d'un lessivage de $\mathrm{K}_{2} \mathrm{O}$.

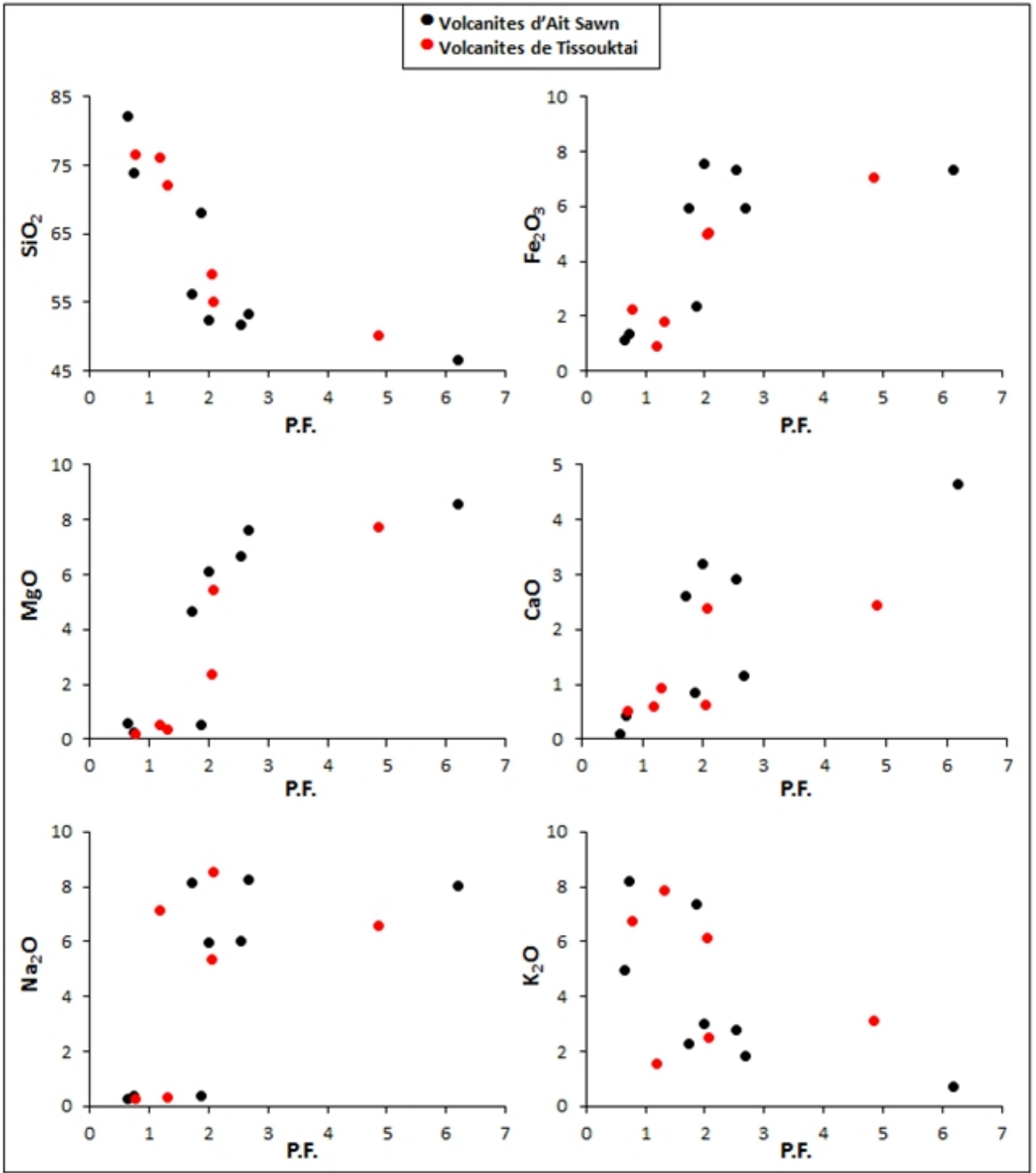

Figure 6 : Distribution des éléments majeurs des roches volcaniques en fonction de la perte au feu 


\section{Minéralisations}

\section{Distribution des minéralisations}

Les minéralisations associées aux roches étudiées peuvent être classées en deux types :

- $\quad$ Une minéralisation disséminée représentée essentiellement par de la malachite qui est associée spatialement au grand filon de quartz de Tissouktai. Ce carbonate, s'associe parfois aux hydroxyles de cuivre (chrysocolle) dans les veines et veinules colmatant les structures réticulées. L'étude métallographique a permis de mettre en évidence deux paragenèses (i) une paragenèse typiquement secondaire constituées de chalcocite, covellite (Planche 2.d) et pyrite disséminée dans la gangue, les sulfures de la paragenèse primaire sont totalement pseudomorphosés ; (ii) une $2^{\text {ème }}$ association plus tardive liée à l'altération superficielle formée de malachite, chrysocolle et oxydes-hydroxydes de fer (Planche 2.e).

- Une minéralisation filonnienne ferrifère associée aux filons subméridiens de quartz, et ceux de barytine orientés N60. Ce type de minéralisation a été identifié dans les deux secteurs d'étude. L'oligiste présente sous forme de sections fibroradiées, tordues ou en baguettes (Planche 2. f).

\section{Mode de dépôt et âge de la minéralisation}

La grande partie des minéralisations cuprifères de l'Anti-Atlas présente un lien génétique étroit avec le magmatisme fini-protérozoïque et plus particulièrement avec le volcanisme édiacarien (Chaabane et Boyer, 1979 ; Zahour, 2001). L'origine, l'âge et la précipitation de ces minéralisations ont fait l'objet de plusieurs travaux de recherche dont les interprétations sont diverses et controversées.

Dans la boutonnière de Tissouktai, la position des laves basiques fortement altérées juste au voisinage du filon de quartz minéralisé en malachite, suggère que le cuivre proviendrait du lessivage des laves basiques. Ces dernières sont recoupées tardivement par des laves acides (ignimbrites et rhyolites de Tissouktai). Par conséquent il serait probable que la minéralisation associée au filon de Tissouktai soit liée au stade hydrothermal contemporain à la mise en place du volcanisme acide associé à l'extension fini-protérozoïque. Or le filon de quartz à malachite marque aussi le contact entre l'édiacarien et la couverture cambrienne et adoudounienne. Il est donc très probable que cette minéralisation soit manifestement liée à un hydrothermalisme post-cambrien, vraisemblablement hercynien

Il en est de même pour les filons d'oligiste/hématite et barytine. L'oligiste serait lié à une phase d'hydrothermalisme responsable de lessivage des oxydes de fer incorporés dans les laves basiques et intermédiaires des volcanites édiacariennes étudiées. 
Les filons minéralisées en barytine et oligiste de direction N60 auraient été liés à une phase hydrothermale plutôt contemporaine à la réactivation des failles N60 pendant l’Hercynien qu’à un hydrothermalisme fini-protérozoïque.

Il faut signaler que Abia et Soulaimani (2012) ont interprété l’oligiste associé à des roches identiques dans l'Anti-Atlas central à des fluides tardifs, probablement suite à une phase d'extension tardi-hercynienne voir triasique.

Faut il noter aussi que les filons de barytine associée aux oxydes de fer qui sont encaissés dans les terrains paléozoïques de la Meseta marocaine ont été interprétés comme produit de remplissages des fractures liées à une phase d'extension NW-SE syn à post-triasique voire jurassique (El Hnot, 2002).

Le cycle hydrothermal aurait été bouclé par une phase supergène plus tardive à malachite, chrysocole, oxydes et hydroxydes de fer.

\section{Contrôle tectonique et âge de la minéralisation}

Les déformations enregistrées par les terrains édiacariens du secteur, sont la conséquence d'une tectonique cassante représentée par des accidents pour la plupart de grande ampleur avec des déplacements considérables. Ces accidents ont déplacé la série étudiée au contact de la série cambrienne et adoudounienne. Ces failles représenteraient probablement un héritage structural du socle panafricain (Azizi Samir, 1990 ; Zahour, 2001) ayant rejoué pendant l'Ediacarien et sont responsables de la mise en place des magmas interprétés en terme d'extension continentale post-panafricaine (Soulaimani et al., 2003), et par conséquent, la circulation des fluides hydrothermaux lors de leur réactivation tardi à post-magmatique. A l'échelle des secteurs étudiés, les failles sont subdivisées en deux générations populations :

- $\quad$ la première génération a une orientation méridienne à sub-équatoriale (N60) et comporte deux familles de failles :

(1) une famille N60 comportant plusieurs failles d'importance cartographique qui ont joué un rôle majeur dans l'évolution paléogéographique de cette zone (Figure 1b). C'est le cas de la faille de Tiflit qui met en contact les schistes et carbonates du Cambrien avec la série volcanique d'Ait Sawan. Un autre exemple est celui de la faille de Tissouktai qui est soulignée par un puissant filon quartzeux $(2 \mathrm{~km} / 10 \mathrm{~m})$ à $\mathrm{Cu}$ et oxydes de fer. Ce dernier souligne le contact entre la série volcanique édiacrienne de Tissouktai et la couverture cambrienne et adoudounienne.

La relation de ces failles avec les formations géologiques étudiées indique donc un rejeu postérieurement à l'époque cambrienne. Ceci suggère que leur remplissage minéralogique serait lié à un événement hydrothermal probablement hercynien et/ou atlasique ? 
Les travaux de Belkacem (2014) sur des terrains de même âge de la vallée de Tifnoute ont permis d'aboutir aux mm résultat.

Cette famille de failles se manifeste aussi par un réseau de fractures colmatées par l'association barytine-oligiste ou par l'oligiste seul. Cette minéralisation peut être liée à un hydrothermalisme qui peut être, soit syn à tardi-magmatique édiacarien, soit contemporain à la réactivation des failles N60 probablement lors de l'Hercynien et/ou l'Atlasique. Les structures tectoniques de la génération des failles sub-équatoriales recoupent les terrains édiacariens des secteurs étudiés et semblent être héritées du socle panafricain. Cependant, elles auraient rejoué lors d'un amincissement crustal contemporain à l'époque édiacarienne favorisant ainsi l'ascension des magmas acides comme en témoigne, entre autres, la fluidalité verticale orienté N60 mise en évidence dans les faciès basiques d'Ait Sawn que dans les ignimbrites d'Ait Sawn et de Tissouktai.

(2) la seconde famille, présente une direction méridienne (NS à N20) est bien développée au niveau de Tissouktai où elle guide les filons du quartz à oligiste, barytine et hématite. Par ailleurs, cette famille de failles aurait guidé les filons rhyolitiques au niveau de Tissouktai. Il est probable donc que ces linéaments tectoniques soient donc déjà actifs pendant l'Ediacarien. Selon cette hypothèse, on pense que les minéralisations contrôlées par cette famille de faille sont liées à un stade hydrothermal qui est peut être soit syn à tardi magmatique édiacarien, soit contemporain à la réactivation de ces structures lors des évenements tectoniques post-cambriens probablement hercyniens.

- $\quad$ La seconde génération de faille est facilement identifiable malgré sa rareté sur le terrain. Elle est scindée en deux familles :

(1) la première est orientée $\mathrm{N} 140$ et elle aurait contrôlé le magmatisme de l'extrusion doméenne d'Ait Sawn (Zahour et al., 2014). Cette génération correspond à celle décrite au niveau d'Imiter (Leistel et Qadrouci, 1991 ; Ouguir et al., 1994 ; Levresse et al., 2000 et Beneddaman, 2014). Des filonets de quartz guidés par les structures de cette génération sont observés sur le terrain.

(2) la deuxième famille est orientée N170 qui a été empruntée par les fluides responsables de la précipitation des filons quartzeux à oligiste. La mise en place de ces filons peut être interprétée de deux manières : (i) soit il s'agit d'une seconde génération de filons de quartz à oligiste liés à un hydrothermalisme syn à tardi-édiacarien; (ii) soit que ces filons seraient contemporains à un hydrothermalisme lié à une ouverture en pull-apart sur décrochement senestre de la génération N60 comme en témoigne l'allure sigmoïdale de ces filons. Un exemple de ces structures est enregistré pendant le Viséen supérieur dans le bassin de Mechraaâ Ben Abbou (Remmal et al., 1997). Cette dernière hypothèse permet d'écarter l'hydrothermalisme 
contemporain aux magmatismes fini-Protérozoïque du secteur, et appuie par conséquent l'origine plus tardive de ces filons.

\section{Conclusion}

Les formations édiacariennes des boutonnières de Tissouktai et d'Ait Sawn sont formées principalement de roches volcaniques (basaltes, andésites basaltiques, andésites, ignimbrites, rhyolites) intercalées localement par des roches volcanoclastiques (brèches pyroclastiques, grès et conglomérats). A cet ensemble sont associés des filons acides qui représenteraient des voies d’alimentation du volcanisme acide. Les paléovolcanites étudiées enregistrent encore l'empreinte des phénomènes d'altération à différents échelles d'observation :

- A l'échelle d'affleurement et de l'échantillon, les phénomènes d'altération présentent une minéralogie et des habitus très variées et marqués par l'apparition des filons, filonets et veines de quartz, oligiste, épidote, barytine, malachite et chrysocole; des vacuoles de feldspath, chlorite, calcite et quartz ; des géodes de quartz et des sphérolites de calcédoine et quartz.

A l'échelle microscopique, les altérations se développent, aussi bien au niveau de la mésostase qui inclut localement des vacuoles millimétriques colmatées par le feldspath, quartz et calcite, qu’aux dépens de minéraux primaires. Dans ce dernier cas, les produits d'altération sont marqués par la présence de chlorite, calcite, quartz, épidote, albite, séricite et oxydes de fer.

A l'échelle géochimique, les altérations se traduisent par un changement dans le comportement des éléments chimiques qui témoigne de l'interaction entre les fluides hydrothermaux et les roches encaissantes. Les diagrammes de variation des oxydes en fonction des pertes au feu montrent un lessivage de $\mathrm{SiO}_{2}$ et de $\mathrm{K}_{2} \mathrm{O}$ et d'un apport de $\mathrm{CaO}, \mathrm{Fe}_{2} \mathrm{O}_{3}, \mathrm{MgO}$ et à moindre mesure $\mathrm{Na}_{2} \mathrm{O}$.

Les caractères pétrographiques, géochimiques, tectoniques et métallogéniques des roches étudiées nous permettent de proposer les évènements hydrothermaux suivants pour expliquer les processus d’altération ayant affecté les paléovolcanites étudiées :

- Un hydrothermalisme syn à tardi magmatique édiacarien contemporain à une phase d'extension intracontinentale fini-protérozoïque. Ce processus n’est toutefois pas à écarter. La pseudomorphose des ferromagnésiens par la chlorite magnésienne, dont la genèse nécessite des températures assez élevées, est l'un des arguments en faveur de cette hypothèse ;

Un hydrothermalisme post-magmatique édiacarien lié à un épisode de circulation des fluides contemporain à la réactivation d'anciennes structures (Failles N60, Nord-Sud, N140 et N170) vraisemblablement lors de la phase hercynienne voire même atlasique (?). Ce scénario semble être très probable. 
Ce stade hydrothermal se manifeste, entre autres, par une remobilisation et reconcentration du cuivre, de la barytine et des oxydes de fer dans les structures filoniennes et d'une altération très prononcée comme en témoignent, entre autres, la dévitrification de la mésostase, l’altération des plagioclases en séricite, épidote, calcite, ainsi que le remplissage des vacuoles par le feldspath, la silice et la calcite.

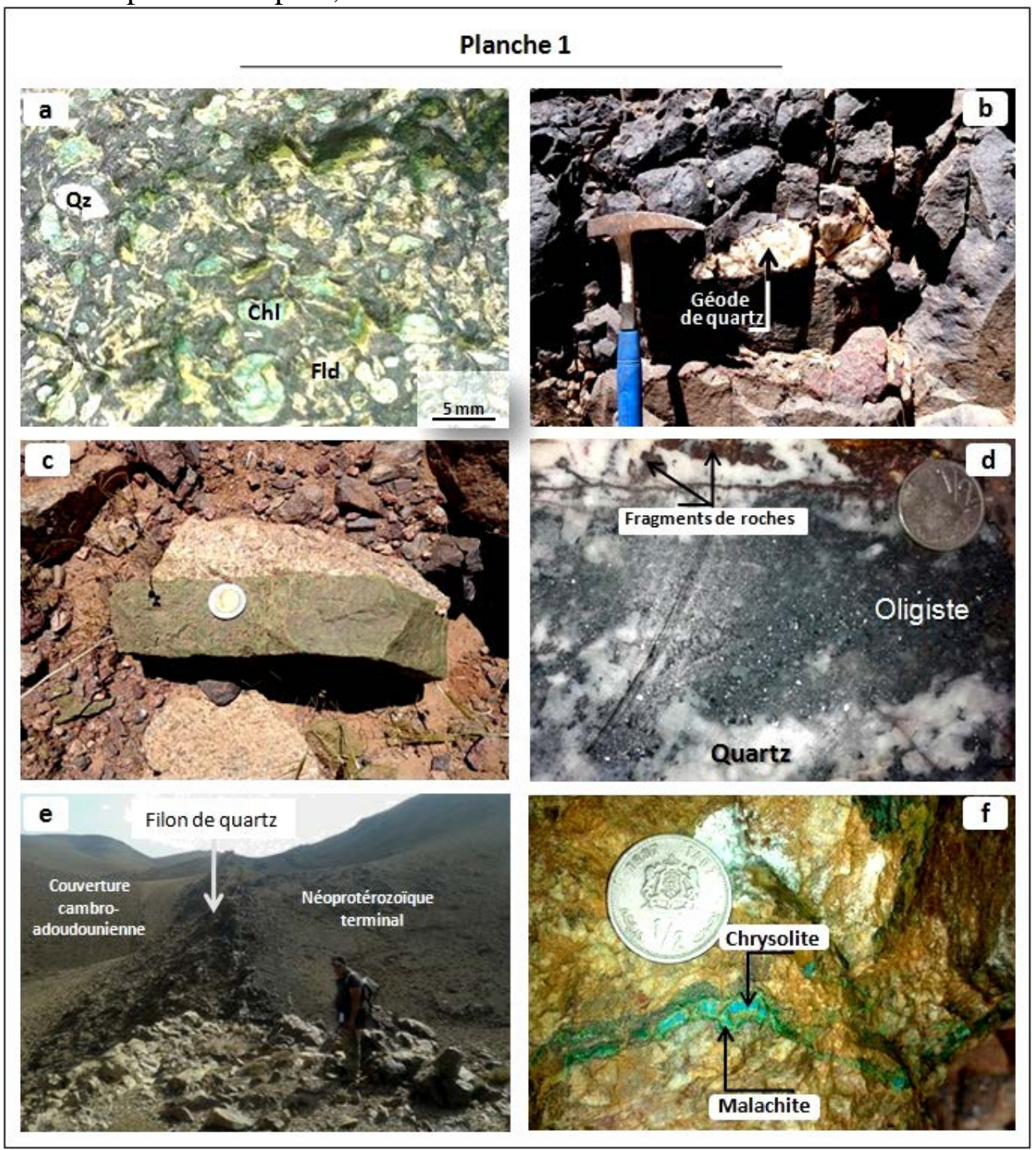

Planche 1 : (a) : Basaltes vacuolaires d'Ait Sawn (Qz :quartz, Chl : chlorite et Fld : feldspath) ; (b) : géode de quartz dans les basaltes sommitaux d'Ait Sawn ; (c) : Filon d'épidote parcourant les basaltes inférieurs d'Ait Sawn ; (d) : Filon de quartz minéralisé en oligiste dans les basaltes de Tissouktai ; (e) : Filon de quartz de Tissouktai marquant le contact anormal entre la série du Néoprotérozoïque terminal et la couverture cambroadoudounienne ; (f) : Veine de malachite et chrysocole parcourant le quartz du filon de Tissouktai. 


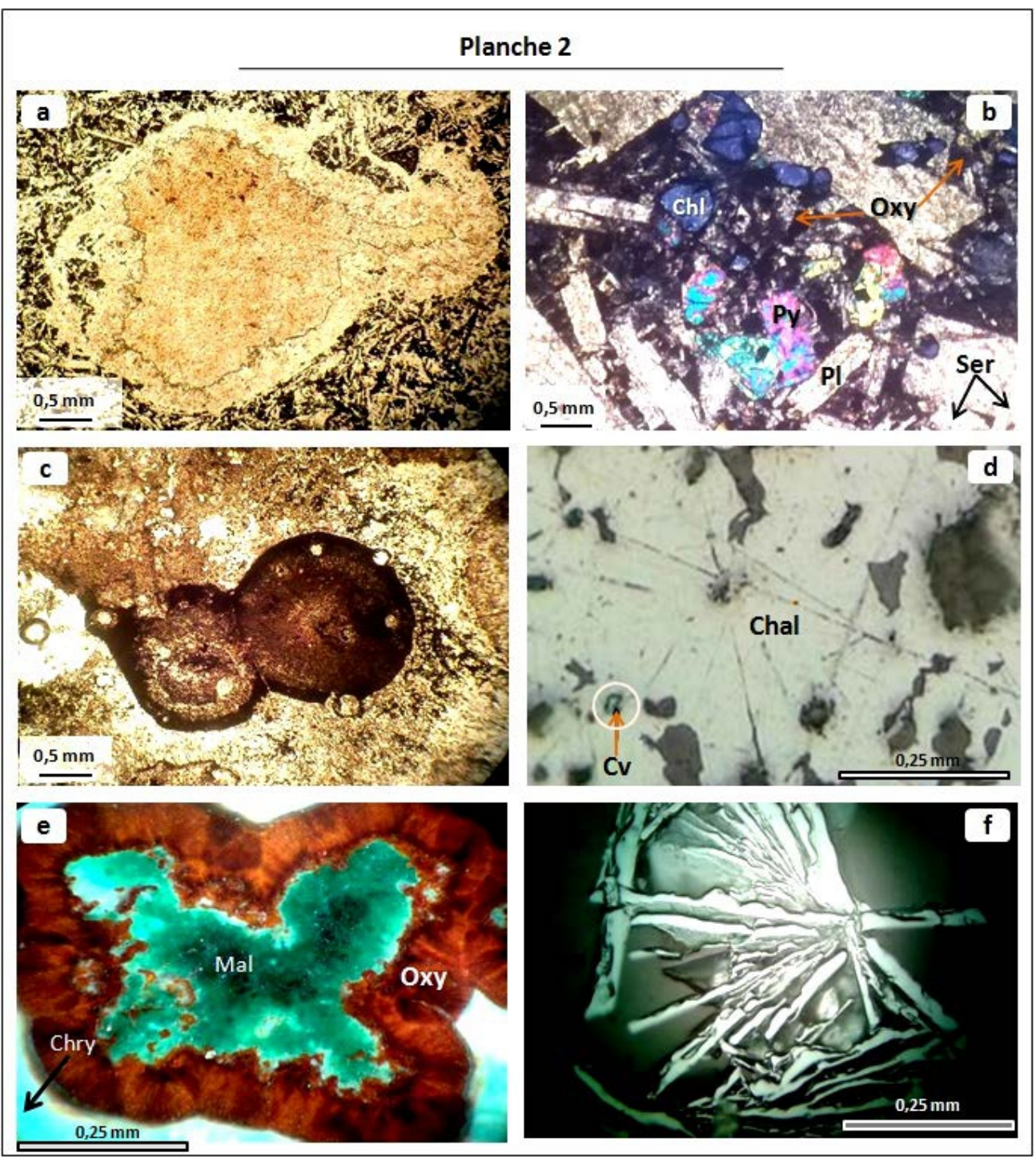

Planche 2 : (a) : Exemple de vacuole dans les basaltes d'Ait Sawn (Fld : Feldspath, Sl : Silice); (b) : aspect microscopique des basaltes à olivine et pyroxène d'Ait Sawn montrant des fantômes d'olivines entièrement altérées en chlorite bleue et oxydes (Chl : chlorite, $\mathrm{Pl}$ : plagioclase, Px : pyroxène, Ser : séricite et oxydes de fer) ; (c) : sphérolites de silice fibroradié dans les ignimbrites d’Ait Sawn ; (d) : Association minéralogique dans le filon de quartz de Tissouktai (Chal : chalcocite et Cv : covellite) ; (e) : Structure colomorphe montrant la paragenèse minéral supergène dans le filon de quartz de Tissouktai (Mal : malachite, Chry : chrysocolle et Oxy : oxyde de fer) ; (f) : Cristaux d'oligiste avec texture fibro-radié 


\section{References:}

Abia E. H. et Soulaiman A. (2012). Les filons de quartz à oligiste de l'AntiAtlas central. Volume 9 (Notes \& Mém. $n^{\circ}$ 564) : Les principales mines du Maroc, pp. 137-149.

Abia El H., Nachit H., Ibhi A. et Baroudi Z. (1999). Les minéralisations filoniennes à $\mathrm{Pb}, \mathrm{Zn}$ et $\mathrm{Cu}$ de la boutonnière de l'ougnat. Relation avec les déformations et essai de calage chronologique. Chron. Rech. Min., 536-537, pp.137-149.

Al Ansari A. et Sagon J.P. (1997). Le gisement d'or de Tiouit (Jbel Saghro, Anti-Atlas, Maroc), un système mésothermal polyphasé à sulfures-or et hématite-or dans une granodiorite potassique d'âge protérozoïque supérieur. Chronique de la Recherche Minière. $\mathrm{N}^{\circ}$ 527, pp.3-25.

Alvaro J. J., Ezzouhairi H., Ait Ayad N., Charif A., Sola R. et Ribeiro M. L (2010). Alkaline lake systems with stromatolitic shorelines in the Ediacaran volcanosedimentary Ouarzazate Supergroup, Anti-Atlas, Morocco. Precambrian Research 179, 22-36.

Azizi-Samir M.R. (1990). Géologie du Précambrien terminal (III) et traitement statistique des données géochimiques de prospection, dans la boutonnière de Bou Azzer- El Graara (Anti-Atlas, Maroc). Thèse Doct. de l' Univ. Joseph Fourier, Grenoble1 : 288 p.

Baroudi Z., Beraaouz E. H., Rahimi A., Saquaque A. et Chouhaidi M. Y. (1999). Minéralisations polymétalliques argentifères d'Imiter (Jbel Saghro, Anti-Atlas, Maroc) : minéralogie, évolution des fluides minéralogiques et mécanismes de dépôts. Chron. Rech. Min., 536-537, pp.91-111.

Baroudi Z. (2002). Le gisement Ag-Hg d'Imiter (Anti-Atlas Oriental) : minéralisation, fluiudes associés et conditions de dépôt. Thèse Doct. d'Etat, Univ. Hassan II Mohammedia, Casablanca (Maroc) : 277 p.

Belkacim S. (2014).Pétrographie, Géochimie et Isotopes ( $\mathrm{Sr}-\mathrm{Nd})$ du volcanisme édiacarien et des dykes doléritiques de la vallée de Tifnoute et caractérisation des indices de $\mathrm{Cu}-\mathrm{Mo} \pm \mathrm{Au}$ d'Imourkhssan (Siroua, Anti-Atlas central, Maroc). Thèse Doct. Naionalt, Univ. Ibn Zohr,Agadir (Maroc): 210 p.

Beneddaman H. (2014). Caractérisation pétrographie et structurale des roches filoniennes et minéralisations associées du Néoprotérozoïque terminal (Précambrien III) d'Imiter Ouest. Mémoire, Université Hassan IIMohammedia Facultés des sciences Ben M’sik Casablanca, Maroc. 25-31p.

Chaabane, A. et Boyer, C., (1979). Séries volcaniques et minéralisations cuprifères du Précambrien supérieur de Tanguerfa, Anti-Atlas, Maroc. C. R. Acad. Sci. Paris, 288, Série D, 5-8.

Choubert G. (1963). Histoire géologique du Précambrien de l'Anti-Atlas. Notes et Mém. Serv. Géol. Maroc, 100 : 75-194. 
En-naciri A., Barbanson L. et Touray J. C. (1997)- Brine inclusions from the Co-As (Au) Bou Azzer district, Anti-Atlas Mountains, Morocco. Econ. Geol., 92, 360-367.

El Hnot H. et Zahraoui M. (2002). Origine et mode de mise en place de la barytine du gisement de Bou Ouzzal (Meseta marocaine. Bulletin de l'Institut Scientifique, Rabat, section Sciences de la Terre, 2005, n²7, 47-54.

Essarraj S., Boiron M. C., Cathelineau M., Banks D. A., El Boukhari A. et Chouhaidi M. Y. (1998). Brines related to Ag deposition in the Zgounder silver deposit (Anti-Atlas, Morocco). Eur. J. Miner., 10, 1201-1214.

Fawcett, J. J. et Yoder H. S. (1966). Phase relationships ofchlorites in the system MgO-Al2O3-SiO2-H2O. Am. Mineral. 51,353-380.

Gasquet, D. et Cheilletz, A., (2009). L'hydrothermalisme : un phénomène cyclique dans les temps géologiques. Conséquences pour la prospection minière au Maroc. in : Stéphanie, G., Christophe, G., (eds.), l'hydrothermalisme en domaine continental. Fonctionnement, ressource et patrimoine, Collection EDYTEM, n 9, 49-58.

Gasquet D., Levresse G., Cheilletz A., Azizi-Samir M.R. et Mouttaqi A., (2005).Contribution to ageodynamic reconstruction of the Anti-Atlas (Morocco) during Pan-African times with the emphasis on inversion tectonics and metallogenic activity at the Precambrien - cambrien transition. Precambrian Res., 140, 157-182.

Giggenbach W F. (1989). Processes controlling $\mathrm{CO}_{2^{-}}$and $\mathrm{Cl}$ - contents of thermal discharges from the Taupo- Rotora volcanic - magmatic hydrothermal system, New Zealand. Proc. Sixth Int. Symp. On Water - Rock Interaction: Rotherdam, Miles D. I. Ed, 259-262.

Leblanc M. (1986). Appareil ignimbritique et minéralisation cuprifère : Alous (Anti-Atlas, Maroc). Mineral. Deposita, 21 : 129-136.

Levresse G., Cheilletz A., Gasquet D., Madi A. et Ait Haddouch L. (2001). Existence de mineralisations Co-Ni d'age norien (Trias superieur) a Bou Azzer (Anti-Atlas, Maroc) : Nouvelles donnees mineralogiques, geochimiques et geochronologiques. In : Proceeding of Magmatisme, Metamorphisme et Mineralisation Associees (3MA), Marrakech.

Liou J. G. (1973). Synthesis and stability relations of epidote, $\mathrm{Ca}_{2} \mathrm{Al}_{2} \mathrm{FeSi}_{3}$ $\mathrm{O}_{12}(\mathrm{OH})$. Journal of Petrology, 14, 381-413.

Leistel J. M. et Qadrouci A. (1991)- Le gisement argentifère d'Imiter (protérozoïque supérieur de l'Anti-Atlas, Maroc). Contrôles des minéralisations, hypothèse génétique et perspectives pour l'exploration. Chron. Rech. min., n502, pp5-22.

Mifdal A. et Peucat J. (1985). Datation U-Pb et Rb-Sr du volcanisme acide de l'Anti-Atlas marocain et du socle sous-jacent dans la région de Ouarzazate. Apport au problème de la limite Précambrien-Cambrien. Sciences Géologiques, Bull., 38 (2) : 185-200. 
Mouttaqi A. et Sagon J. P. (1999). Le gisement de cuivre de Bleïda (AntiAtlas central): une interférence entre les processus de remplacement et d’exhalaison dans un contexte de rift. Chron. Rech. Min., 536-537, pp.5-21.

Norton D. (1978).Sourcelines, sourceregions, and pathlines for fluids in hydrothermal systems related to cooling plutons. Economic Geology, 73, pp. 21-28.

Ouguir H., Macaudiere J., Dagallier G., Qadroucia. et Leistel J.M. (1994). Cadre structural du gite Ag-Hg d'Imiter (Anti-Atlas, Maroc) : implication metallogenique. Bull. Soc. Geol., Fr., 165, 233-248.

Plumb K.A. (1991) - New Precambrian times scale. Episodes, Vol. 14, $\mathrm{n}^{\circ} 2$, pp. 139-140.

Remmal T., El Kamel F. et Mohsine A. (1997).Le bassin viséen de Mecharaâ Ben Abbou (Meseta occidentale, Maroc) : une structure sur décrochement $\mathrm{N} 80^{\circ}$ associée à un magmatisme tholéïtique d'intraplaque. Géol. Méd. Tome XXIV n 3-4. 1997. Pp. 225-239.

Rosenbauer R.J, Bischoff J.L. et Zierenberg R.A. (1988). The laboratory albitization of mid-ocean ridge basalt. J. Geol., 96, 237-244.

Soulaimani A., Bouabdelli M. et Piqué A. (2003). L'extension continentale au Néo-Protérozoïque supérieur-Cambrien inférieur dans l'Anti-Atlas (Maroc), Bull. Soc. géol. France, 174 (1) : 83-92.

Thomas R.J., Fekkak A., Ennih N., Errami E., Loughlin S.C., Gresse P.G, Chevallier L.P. et Liegeois J.-P. (2004). A new lithostratigraphic framework for the Anti-Atlas Orogen, Morocco. Jl. Afr. Earth Sci., 39 (3-5): 217-226.

Walsh G.J., Aleinikoff J. N., Benziane F., Yazidi A. et Armstrong T. R. (2002). U-Pb zircon geochronology of the Paleoproterozoic Tagrara de Tata inlier and its Neoproterozoic cover, Western Anti-Atlas, Morocco. Precambrian Research, $117: 1-20$.

Zahour R., Zahour G., SY A. H., Baroudi Z., El Hadi H. et Zerhouni Y. (2014). Le volcanisme domméen du Néoproterozoïque terminal de la boutonnierre d'Ait Sawn (Anti-Atlas oriental, Maroc). Ann. Soc. Géol. du Nord T 21 pp: 51-58.

Zahour, G. (2001). Le Néoprotérozoïque terminal de la boutonnière de Toubkal (Haut-Atlas Occidental) et de Siroua (Anti-Atlas Central) : un exemple de volcanisme intraplaque continental associé à un volcanisme calco-alcalin post-collisionnel. Thèse Doct. d'Etat, Univ. Hassan II Mohammedia, Casablanca (Maroc) : 266 p.

Zahour G. (1990). — Etude du volcanisme Précambrien terminal d'Aït Maghlif (Siroua Nord-Est, Anti-Atlas central, Maroc). Lithostratigraphie Pétrographie-Géochimie. Thèse de spécialité 3e cycle, Univ. Cadi Ayyad, Marrakech (Maroc) : 236 p. 